\title{
Apple Watch, Wearables, and Heart Rhythm: where do we stand?
}

\author{
Joel M. Raja ${ }^{1}$, Carol Elsakr ${ }^{2}$, Sherif Roman ${ }^{3}$, Brandon Cave ${ }^{4}$, Issa Pour-Ghaz ${ }^{1}$, Amit Nanda ${ }^{1}$, \\ Miguel Maturana ${ }^{1}$, Rami N. Khouzam ${ }^{5}$
}

${ }^{1}$ Department of Internal Medicine, University of Tennessee Health Science Center, Memphis, TN, USA; ${ }^{2}$ Department of Medicine, The George Washington University School of Medicine \& Health Sciences, Washington, DC, USA; ${ }^{3}$ Department of Medicine, Cairo University, Cairo, Egypt; ${ }^{4}$ Department of Pharmacy, Methodist University Hospital, Memphis, TN, USA; ${ }^{5}$ Department of Internal Medicine, Division of Cardiovascular Diseases, University of Tennessee Health Science Center, Memphis, TN, USA

Contributions: (I) Conception and design: JM Raja, RN Khouzam; (II) Administrative support: JM Raja, RN Khouzam; (III) Provision of study materials or patients: All authors; (IV) Collection and assembly of data: JM Raja, C Elsakr, S Roman, B Cave, I Pour-Ghaz, A Nanda, M Maturana; (V) Data analysis and interpretation: JM Raja, C Elsakr, S Roman, B Cave, I Pour-Ghaz, A Nanda, M Maturana; (VI) Manuscript writing: All authors; (VII) Final approval of manuscript: All authors.

Correspondence to: Joel M. Raja, MD. Department of Internal Medicine, University of Tennessee Health Science Center, 2750 Mcvay Road, Memphis, TN 38119, USA. Email: jraja@uthsc.edu.

\begin{abstract}
Atrial fibrillation (AF) poses a major health concern in the United States by affecting over 5 million people accounting for at least $15 \%$ to $25 \%$ of strokes. It can be asymptomatic or subclinical with its first presentation being stroke in $18 \%$, and AF being only detected at the time of stroke. With evidence of subclinical AF associated with increased risk of ischemic stroke, recent developments indeed point towards wearables, especially smart watches, being quite effective and representing a novel method for screening for silent AF in the general population, and thereby reducing mortality and morbidity associated with it. This manuscript aims to review whether the photoplethysmography (PPG) technology, employed in the wearables to monitor heart rate, is accurate enough to aid in the diagnosis of AF that may remain asymptomatic or paroxysmal. It also explores the option of actually employing this method in the general population, the feasibility of this mode of diagnosis, sensitivity and specificity of this method compared to the conventional electrocardiogram (EKG), and the actual follow up with a practitioner and subsequent treatment of AF, if diagnosed. We conducted a Medline search using various combinations of "smart watch" "atrial fibrillation" "wearables", and "Kardia" to identify pivotal randomized trials published before June 1, 2019, for inclusion in this review. Concurrently, major practice guidelines, trial bibliographies, and pertinent reviews were examined to ensure inclusion of relevant trials. A consensus among the authors was used to choose items for narrative inclusion. The following section reviews data from pivotal trials to determine the effectiveness of smart watch technology in detecting AF in the general population. Trials reviewed evaluated apple watch, Kardia, Samsung wearables in diagnosis of AF. The fact that there is an increase in consumer use of wearables, smart devices, which can serve as health monitoring devices that can be used as a non-invasive, ambulatory assessment of heart rate and rhythm, is definitely novel. Intermittent short EKG recordings repeated over a longer-term period produced significantly better sensitivity for AF detection, with 4 times as many cases diagnosed compared with a single time-point measurement. Since there are limitations and further research into this new field is required, the wearable technology may not serve as the ultimate tool for diagnosis of AF, rather a nidus for the general population to seek medical advice for confirmation on being notified of having an irregular rhythm leading to prevention of morbidity and mortality associated with it.
\end{abstract}

Keywords: Apple watch; wearables; single lead electrocardiogram (single lead EKG); atrial fibrillation (AF); arrhythmia

Submitted May 29, 2019. Accepted for publication Jun 23, 2019.

doi: 10.21037/atm.2019.06.79

View this article at: http://dx.doi.org/10.21037/atm.2019.06.79 


\section{Introduction}

Atrial fibrillation (AF) poses a major health concern in the United States by affecting over 5 million people (1). It is associated with significant mortality and morbidity by increasing the risk of stroke 5 -fold compared to general population (2). In the United States, AF accounts for at least $15 \%$ to $25 \%$ of strokes (3). It can be asymptomatic or subclinical with its first presentation being stroke in $18 \%$, and $\mathrm{AF}$ being newly detected at the time of stroke (4). Furthermore, the prevalence of AF increases with age, being 65 or older places the prevalence around $4.4 \%$ with people who have previously undiagnosed AF making $1.4 \%$ of it (5). Estimates of people in the United States who may have previously unknown, asymptomatic $\mathrm{AF}$ are around 700,000 , with an estimated cost burden of 3.2 billion dollars $(6,7)$.

With evidence of subclinical AF associated with increased risk of ischemic stroke (8), previously only being detected by ambulatory electrocardiogram (EKG) monitoring either with temporary patches for short periods of time or implantable recorders for longer periods, there might be a place for wearable technology, which is typically used on a regular basis, in aiding diagnosis of subclinical AF. As the popularity of smart watches increases, with more than 325 million devices sold in 2016 alone (9) and $13 \%$ of the US population owning smartwatches, with $40 \%$ of the public showing interest in getting one (10), these devices are becoming a commodity rapidly finding its way on everyone's wrist and in pockets. The use of these wearables needs to be effective in diagnosing subclinical AF, with false positives increasing health costs by unwarranted ER visits or anxiety to the patient, or with false negatives missing the diagnosis which may lead to morbidity or mortality.

Recent developments indeed point towards wearables, especially smart watches, being quite effective and being a novel method for screening for silent $\mathrm{AF}$ in the general population. Though the technology is not only restricted to smart watches, single lead EKGs that are portable and phone applications that detect heart rate can serve as event recorders during an episode of symptoms like palpitations, but may be restricted by not providing continuous monitoring.

\section{Discussion}

In this review, we will discuss the technology behind the wearables and its efficacy. Further implementation of this technology in different formats will also be addressed.

\section{Photoplethysmography (PPG)}

PPG is the measure of the volumetric change of the heart by measuring light transmission or reflection. During systole, the rise in pressure forces a forward pulse into the blood vessel. The device shines light on the skin to detect a pulse by measuring the difference in the quantity of light reflected back to the sensor. The sensor is usually placed on the skin in the areas where the artery is proximal to the skin. Thus, the heart rate is measured using an algorithm. PPG technology can be used in physical sensors, smart watches, or even phone apps to measure heart rate.

PPG which is deemed as medical grade is accurate at measuring heart rate in sinus rhythm (correlation coefficient of 0.96) (11). However, PPG is limited by it underestimation of heart rate during sinus tachycardia and accuracy suffers during physical activity. On testing consumer grade PPG technology (Apple watch, Fitbit, etc.), its accuracy was also high in sinus rhythm (12). A small study analyzing PPG data from smart watches with a deep neural network to detect AF compared to a standard 12 lead EKG yielded a sensitivity of $98.0 \%$ and specificity of $90.2 \%$ (13). This proves the data obtained by PPG technology in the wearables when analyzed by algorithms can be effective in diagnosing AF.

Implementation of the PPG technology through certain apps in phones can also be used to measure heart rate. With $77 \%$ of the US population owning a smartphone (14), this can be implemented as a measure to detect irregular heart rate in general population. Cardiio Rhythm app for I-phone implemented the phone's camera as a light sensor to obtain heart rate measurements. An outpatient study involving 1,013 participants with hypertension, diabetes mellitus, and/or aged $\geq 65$ years, revealed that the app was able to detect Atrial fibrillation with a sensitivity of $92.9 \%$ (95\% CI: 77-99\%), specificity of $97.7 \%$ (95\% CI: $97-99 \%$ ), with a positive predictive value (PPV) of $53.1 \%$ (95\% CI: 38-67\%), and a negative predictive value (NPV) of 99.8\% (95\% CI: 99-100\%) (15). Although this app cannot get continuous heart rate data from the person, random heart rate checks can uncover subclinical AF or even during symptoms palpitations act like an event recorder to pick up an episode of AF.

\section{Apple watch study}

The Apple watch study was a prospective, single arm, open label study with 419,297 participants conducted with a goal of evaluating the ability of irregular pulse detection by the 
Apple watch and subsequent irregular pulse notification algorithm to identify AF (16). The primary endpoint was $\mathrm{AF}>30$ seconds on EKG patch and simultaneous AFib on EKG patch and tachogram. The heart rate is recorded as PPG waveforms by the Apple watch during periods of minimal arm movement. Tachograms, which are heart rate over a period of time, are created. If a tachogram detects irregular heart rate, then the algorithm is triggered to get 4 confirmatory tachograms with irregular pulse rates during minimal arm movements. After 4 confirmatory tachograms, 5 in total including the initial irregular tachogram, the participant is alerted through a phone notification. The notification encourages the participant to connect to a Telehealth doctor, who based on the data from the Apple watch readings and symptoms will advise the patient to go to the $\mathrm{ED}$ if he deems the patient requires immediate medical attention or will recommend an EKG patch that will be shipped to the participant to be worn concurrently with the watch for up to 7 days and returned at the end of the study period for analysis.

The study population consisted of people aged $\geq 22$ years without history of AF or atrial flutter and current use of anticoagulation. Of the study population, the mean age was 41 with the age group of 22 to 39 years old making $52 \%$ of the study population. About 2,161 people got notified of irregular heart rate and 658 of them ended up getting the EKG patch with only 450 returned for analysis. Notification rate was highest among the older population $(\geq 65)$. AF was detected in $34 \%$ of the cohort, who received a notification and wore the EKG patch. This does not negate the fact that more people may have been diagnosed with AFib with longer EKG patch monitoring. Based on the analysis of the PPG technology used in the Apple watch validated by the concurrent use of the EKG patch, which is the current standard for diagnosing paroxysmal arrhythmias, the PPV of the tachograms was found to be 0.71 (95\% CI: 0.69-0.74), and the PPV of notifications, triggered by 5 tachograms, was found to be 0.84 (95\% CI: 0.76-0.92).

Although the study shows Apple watch might be a viable initial diagnostic tool in subclinical $\mathrm{AF}$, the majority of the cohort was made up of young participants with the study failing to reach the target enrollment of 75,000 aged 65 or older. The population was also relatively healthier with $21 \%$ having hypertension, $5 \%$ having diabetes mellitus. The study also relied on self-reporting of the patients who are presented with the notification, though the post study survey reveals that $57 \%$ of the study population sought medical help outside of the Tele-health system implemented in the study, of which $36 \%$ underwent additional testing, with $33 \%$ being referred to a specialist and $28 \%$ being started on a new medication. This can be a sign that majority did indeed take the notification seriously to get it further investigated by a physician.

\section{Kardia Band (KB)}

$\mathrm{KB}$ is a product made by AliveCor designed to be used as an Apple Watch accessory. It mimics lead I and can record a rhythm strip for $30 \mathrm{~s}$. The KB application can then use an algorithm to detect whether the strip shows atrial fibrillation (AF) or not. Bumgarner et al. (17) conducted a single center, prospective, nonrandomized, adjudicatorblinded study with 100 participants to assess whether $\mathrm{KB}$ algorithms could distinguish AF from sinus rhythm as well as electrophysiologist-interpreted 12-lead EKGs can. The three labels the $\mathrm{KB}$ algorithm can give a rhythm strip are "possible AF" (based on rhythm irregularity and P-wave absence), "normal" (no AF detected and heart rate is $50-100$ beats/min), and "unclassified" (no AF detected and heart rate is $<50$ or $>100$ beats/min or recording is either noisy or $>30$ seconds, making up $34 \%$ of the recordings). The study population consisted of people aged 18-90 years with a history of AF who presented for a scheduled elective electrical cardioversion. Of the study population, the mean age was 68; 169 simultaneous $\mathrm{KB}$ and EKG recordings were made of each patient before and after cardioversion; 15 patients ended up not undergoing cardioversion with 8 of them cancelling due to being in sinus rhythm rather than $\mathrm{AF}$.

Among the positive readings, the $\mathrm{KB}$ algorithm correctly detected AF with $93 \%$ sensitivity (95\% CI: 86-99\%), 84\% specificity (95\% CI: 73-95\%), and k coefficient of 0.77 (95\% CI: 0.65-0.89) when compared to electrophysiologistinterpreted 12-lead EKG recordings. Electrophysiologists who examined all 169 of the $\mathrm{KB}$ recordings (in total, they could interpret 147; 22 of them were non-interpretable due mainly to baseline artifact) correctly detected AF with $99 \%$ sensitivity (95\% CI: $96-100 \%$ ), $83 \%$ specificity (95\% CI: $74-92 \%$ ), and $k$ coefficient of 0.83 (95\% CI: $0.74-$ 0.92 ) when compared to electrophysiologist-interpreted 12-lead EKG recordings. Thus, the study demonstrates that he $\mathrm{KB}$ algorithm is pretty effective in detecting $\mathrm{AF}$, with increase in sensitivity when supported by physician review.

However, the authors note limitations of this study such as the small sample size and patients all having AF significant enough to be scheduled for electric cardioversion. Generalizability may also be limited due to 
participants being $17 \%$ female, socioeconomic status not being examined, patients with implanted pacemakers or defibrillators being excluded, and participants never having used KB before. There could have been less unclassified readings if participants had more practice with $\mathrm{KB}$, but $\mathrm{KB}$ performance may decrease outside these study settings as the participants were taught how to use KB immediately before each supervised recording. The study including patients with already known $\mathrm{AF}$ limits it from commenting about its efficiency in detecting subclinical AF.

\section{Handheld EKG devices}

Another way Smartphones can be used as EKG monitors is by interfacing with peripherals such as a special smartphone case with embedded electrodes to acquire, store, and transfer single-channel EKG rhythms like AliveCor Heart Monitor (AHM), which has already been US Food and Drug Administration cleared and Conformite Europeenne (CE) marked, and My Diagnostick handheld EKG stick.

The Chan et al. outpatient study also analyzed singlelead EKG recorded by using the AliveCor heart monitor with tracings reviewed subsequently by 2 cardiologists to provide the reference standard in which Patients with hypertension, with diabetes mellitus, and/or aged $\geq 65$ years were recruited. The study found that The diagnostic sensitivity of the AliveCor automated algorithm 71.4\% (95\% CI: 51-87\%), the specificity $99.4 \%$ (95\% CI: $99-100 \%$ ), the PPV $76.9 \%$ (95\% CI: $56-91 \%)$, NPV 99.2\% (95\% CI: 98-100\%) (15).

To determine the usability, accuracy, and costeffectiveness of two handheld single-EKG devices for AF screening in a hospital population; a study was conducted in which 445 hospitalized patients at cardiological or geriatric wards were screened for AF by two handheld EKG devices (My Diagnostick and AliveCor) (18). The performance of the automated algorithm of each device was evaluated against a full 12-leador 6-lead EKG recording. After the exclusion of patients with an implanted device, sensitivity and specificity of the automated algorithms were suboptimal (Cardiology: $81.8 \%$ and $94.2 \%$, respectively, for MyDiagnostick; $54.5 \%$ and $97.5 \%$, respectively, for AliveCor) (Geriatrics: $89.5 \%$ and $95.7 \%$, respectively, for MyDiagnostick; $78.9 \%$ and $97.9 \%$, respectively, for AliveCor).

Manual interpretation increases sensitivity, but decreases specificity, doubling the cost per detected patient, but remains cheaper than sole 12-lead EKG screening.

The accuracy of blinded clinician interpretation of single-lead smartphone electrocardiograms was investigated by conducting a prospective, blinded, observational cohort study in which Consecutive patients 18 years and older undergoing electrical cardioversions for $\mathrm{AF}$ and atrial flutter were recruited over 12 months (19). AHM was paired with an iPhone 6-Plus smartphone using the Kardia application. Three consecutive 30-second lead I recordings (iEKG) were obtained with finger placement on the 2 electrodes at the back of the iPhone. Following each AHM recording, the automated rhythm analysis recorded the rhythm as either possible AF, normal sinus rhythm, or unclassified. A 12-lead EKG was obtained prior to the iEKG tracings, both preand post- cardioversion. On assessing the accuracy of AHM on all tracings, including "unclassified" readings (marked as incorrect), the AHM demonstrated reduced sensitivity $(77 \%)$ and specificity (76\%).

These devices receive most interest for opportunistic screening, as they are easy to use, portable, low-cost, allow fast rhythm strip recordings, do not require experienced personnel, and often have built-in algorithms that provide an immediate interpretation of the EKG. There are also limitations for their use. The majority of false positives results originated from pulse waveforms that were corrupted by finger movement artifacts that may have affected the detection algorithm. This issue may lead to a reduction in specificity when the smartphone application is used outside the clinic because of potentially more motion artifacts in an unsupervised setting, e.g., using it repeatedly at home over a period of weeks or even longer. A high number of false positives could create additional work for clinicians to rule out AF. Another drawback of the application is the inability to detect atrial flutter that may also confer some risk of stroke and frequently accompanies AF.

\section{Conclusions}

The fact that there is a significant increase in consumer use of wearables, smart watches; which can serve as health monitoring devices that can be used as a non-invasive, ambulatory assessment of heart rate and rhythm, is definitely novel. The different wearable technology and its efficacy in detecting AF (Table 1) is well demonstrated in different studies. The demonstration of majority of the study population in the apple study seeking medical help after being notified is encouraging. On the flip side, false positives can cause undue anxiety to the user and may drive up health costs with unwarranted ER visits. The system of irregular rhythm notification in people without a known 
Table 1 Sensitivity, specificity, PPV, and NPV of Smart Watch, Wearables, and Handheld EKG devices in detecting AF

\begin{tabular}{|c|c|c|}
\hline Smart Watch/Wearable/Handheld EKG & Studies & Sensitivity/specificity/PPV/NPV \\
\hline Kardia Band (FDA approved) & Bumgarner et al., 2018 & Sensitivity: $93 \%$; specificity: $84 \%$ \\
\hline \multirow{3}{*}{ Alive Cor single lead EKG (FDA approved) } & \multirow[t]{2}{*}{ Desteghe et al., 2016} & Cardiology ward: sensitivity: $54.5 \%$; specificity: $97.5 \%$ \\
\hline & & Geriatrics ward: sensitivity: $78.9 \%$; specificity: $97.9 \%$ \\
\hline & Koshy et al., 2018 & Sensitivity: 77\%; specificity: 76\% \\
\hline My Diagnostick & Desteghe et al., 2016 & Cardiology ward: sensitivity: $81.8 \%$; specificity: $94.2 \%$ \\
\hline
\end{tabular}

PPV, positive predictive value; NPV, negative predictive value; EKG, electrocardiogram; FDA, Food and Drug Administration.

diagnosis of AF supported by physician review can help in uncovering the subclinical AF population.

Recent evidence shows that intermittent short EKG recordings repeated over a longer-term period produced significantly better sensitivity for AF detection, with 4 times as many cases diagnosed compared with a single time-point measurement (20). This further reiterates the fact that these wearables serve as an excellent resource for continuous EKG monitoring with data saved in the cloud which can be assessed from anywhere, as compared to other devices as Holter monitors and event monitors, limited by the intermittent nature of monitoring and the need to be returned for analysis. In addition, they also are non-invasive methods for prolonged periods of screening compared to the invasive implantable loop recorders. Data also shows that preference of patients lean more towards wearable technology as they are perceived as convenient by $98 \%$, while $90 \%$ conveyed they were likely to use these devices to determine cardiac rhythm during symptomatic episodes as opposed to conventional Holter monitoring system (21).

The technology may however be limited by patient specific barriers, like age. The prevalence of AF markedly increases with age, though older population may be more resistant in adopting these wearable technologies. The cohort in the Apple heart study were overwhelmingly young and healthier. Furthermore, studies are required to adjudicate the effectiveness of the wearables in detecting AF in high risk population. Plus, compared the smart watch technology, where there is an element of passive detection, the smart phone apps and handheld EKG have an active aspect of user participation for recording strips of EKG. Improper use may not yield proper data. Even the smartwatches have to overcome improper data measurements triggered by motion and hand movement, which may yield false positives. The technological shortcomings are bound to get better with more research and development poured into these devices and the detection algorithms. Future iterations of these devices will improve its efficiency to better detect the subclinical AF.

These devices have even shown to be cost-effective when used in a community screening program $(22,23)$ and they can be used for the follow-up assessment of the effectiveness of $\mathrm{AF}$ treatment. Thus, the wearable technology may not serve as the ultimate tool for diagnosis of AF, rather a nidus for the general population to seek medical advice on being notified of having an irregular rhythm for confirmation leading to prevention of morbidity and mortality associated with it.

\section{Acknowledgments}

None.

\section{Footnote}

Conflicts of Interest: The authors have no conflicts of interest to declare.

Ethical Statement: The authors are accountable for all aspects of the work in ensuring that questions related to the accuracy or integrity of any part of the work are 
appropriately investigated and resolved.

\section{References}

1. Miyasaka Y, Barnes ME, Gersh BJ, et al. Secular Trends in Incidence of Atrial Fibrillation in Olmsted County, Minnesota, 1980 to 2000, and Implications on the Projections for Future Prevalence. Circulation 2006;114:119-25.

2. Wolf PA, Abbott RD, Kannel WB. Atrial fibrillation as an independent risk factor for stroke: the Framingham Study. Stroke 1991;22:983-8.

3. Marini C, De Santis F, Sacco S, et al. Contribution of atrial fibrillation to incidence and outcome of ischemic stroke: results from a population-based study. Stroke. 2005;36:1115-9.

4. Lin HJ, Wolf PA, Benjamin EJ, et al. Newly diagnosed atrial fibrillation and acute stroke. The Framingham Study. Stroke 1995;26:1527-30.

5. Lowres N, Neubeck L, Redfern J, et al. Community screening programs to identify unknown atrial fibrillation: a systematic review. Eur Heart J 2012;33:61.

6. Turakhia MP, Shafrin J, Bognar K, et al. Estimated prevalence of undiagnosed atrial fibrillation in the United States. PLoS One 2018;13:e0195088.

7. Turakhia MP, Shafrin J, Bognar K, et al. Economic Burden of Undiagnosed Nonvalvular Atrial Fibrillation in the United States. Am J Cardiol 2015;116:733-9.

8. Healey JS, Connolly SJ, Gold MR, et al. Subclinical atrial fibrillation and the risk of stroke. N Engl J Med 2012;366:120-9.

9. IDC Forecasts Shipments of Wearable Devices to Nearly Double by 2021 as Smart Watches and New Product Categories Gain Traction. Available online: https://www. idc.com/getdoc.jsp? containerId=prUS43408517

10. Lamkin P. Smartwatch popularity booms with fitness trackers on the slide. Forbes. Available online: https://www. forbes.com/sites/paullamkin/2018/02/22/smartwatchpopularity-booms-with-fitness-trackers-on-the-slide

11. Koshy AN, Sajeev JK, Nerlekar N, et al. Utility of photoplethysmography for heart rate estimation among inpatients. Intern Med J 2018;48:587-91.

12. Koshy AN, Sajeev JK, Nerlekar N, et al. Smart watches for heart rate assessment in atrial arrhythmias. Int J Cardiol 2018;266:124-7.

13. Tison GH, Sanchez JM, Ballinger B, et al. Passive Detection of Atrial Fibrillation Using a Commercially Available Smartwatch. JAMA Cardiol 2018;3:409-16.
14. Smith A. Record shares of Americans now own smartphones, have home broadband. Pew Internet \& American Life Project 2017. Available online: https:// www.pewresearch.org/fact-tank/2017/01/12/evolution-oftechnology/

15. Chan PH, Wong CK, Poh YC, et al. Diagnostic Performance of a Smartphone-Based Photoplethysmographic Application for Atrial Fibrillation Screening in a Primary Care Setting. J Am Heart Assoc 2016. doi: 10.1161/jaha.116.003428.

16. Turakhia MP, Desai M, Hedlin H, et al. Rationale and design of a large-scale, app-based study to identify cardiac arrhythmias using a smartwatch: The Apple Heart Study. Am Heart J 2019;207:66-75.

17. Bumgarner J, Lambert C, Cantillon D, et al. Assessing The Accuracy Of An Automated Atrial Fibrillation Detection Algorithm Using Novel Smartwatch Technology Among Patients Presenting For Elective Cardioversion. JACC 2018. doi: 10.1016/S0735-1097(18)30952-5.

18. Desteghe L, Heidbuchel H. 'Performance of handheld electrocardiogram devices to detect atrial fibrillation in a cardiology and geriatric ward setting: authors response.' Europace 2017;19:1408-9.

19. Koshy AN, Sajeev JK, Negishi K, et al. Accuracy of blinded clinician interpretation of single-lead smartphone electrocardiograms and a proposed clinical workflow. Am Heart J 2018;205:149-53.

20. Svennberg E, Engdahl J, Al-Khalili F, et al. Mass Screening for Untreated Atrial Fibrillation: The STROKESTOP Study. Circulation 2015;131:2176-84.

21. Rajakariar K, Koshy A, Sajeev J, et al. Increased preference of wearable devices compared with conventional cardiac monitoring. Heart, Lung Circulation 2018;27:S170.

22. Levin LÅ, Husberg M, Sobocinski PD, et al. A cost-effectiveness analysis of screening for silent atrial fibrillation after ischaemic stroke. Europace 2015;17:207-14.

23. Aronsson M, Svennberg E, Rosenqvist $M$, et al. Costeffectiveness of mass screening for untreated atrial fibrillation using intermittent ECG recording. Europace 2015;17:1023-9.

Cite this article as: Raja JM, Elsakr C, Roman S, Cave B, Pour-Ghaz I, Nanda A, Maturana M, Khouzam RN. Apple Watch, Wearables, and Heart Rhythm: where do we stand? Ann Transl Med 2019;7(17):417. doi: 10.21037/atm.2019.06.79 